\title{
SEARCH FOR VHE $\gamma$-RAY EMISSION FROM THE GLOBULAR CLUSTER M13 WITH THE MAGIC TELESCOPE
}

H. Anderhub ${ }^{1}$, L. A. Antonelli ${ }^{2}$, P. Antoranz ${ }^{3}$, M. Backes ${ }^{4}$, C. Baixeras ${ }^{5}$, S. Balestra ${ }^{3}$, J. A. Barrio ${ }^{3}$, D. Bastieri ${ }^{6}$, J. Becerra González ${ }^{7}$, J. K. Becker ${ }^{4}$, W. BednareK ${ }^{8}$, K. Berger $^{8}$, E. Bernardini ${ }^{9}$, A. Biland ${ }^{1}$, R. K. Bock ${ }^{6,10}$, G. Bonnoli ${ }^{11}$, P. Bordas ${ }^{12}$, D. Borla Tridon ${ }^{10}$, V. Bosch-Ramon ${ }^{12}$, D. Bose ${ }^{3}$, I. Braun ${ }^{1}$, T. Bretz ${ }^{13}$, I. Britvitch ${ }^{1}$, M. Camara ${ }^{3}$, E. Carmona ${ }^{10}$, S. Commichau ${ }^{1}$, J. L. Contreras ${ }^{3}$, J. Cortina ${ }^{14}$, M. T. Costado ${ }^{7}, 15$, S. Covino ${ }^{2}$, V. CurteF ${ }^{4}$, F. Dazzi ${ }^{16,25}$, A. De Angelis ${ }^{16}$, E. De Cea del Pozo ${ }^{17}$, R. de los Reyes ${ }^{3}$, B. De Lotto ${ }^{16}$, M. De Maria ${ }^{16}$, F. De Sabata ${ }^{16}$, C. Delgado Mendez ${ }^{7,26}$, A. Dominguez ${ }^{18}$, D. Dorner ${ }^{1}$, M. Doro $^{6}$, D. Elsaesser ${ }^{13}$, M. Errando $^{14}$, D. Ferenc ${ }^{19}$,

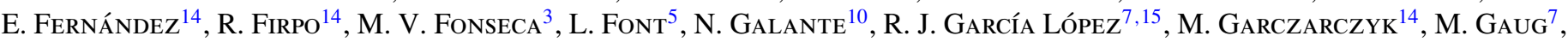
F. Goebel ${ }^{10,27}$, D. Hadasch ${ }^{5}$, M. HAyashida ${ }^{10}$, A. Herrero ${ }^{7,15}$, D. Hildebrand ${ }^{1}$, D. HöHNe-MönCh ${ }^{13}$, J. Hose ${ }^{10}$, C. C. Hsu $^{10}$, T. Jogler ${ }^{10}$, D. Kranich ${ }^{1}$, A. La Barbera ${ }^{2}$, A. LAILle ${ }^{19}$, E. Leonardo ${ }^{11}$, E. LindFors ${ }^{20}$, S. Lombardi ${ }^{6}$, F. Longo ${ }^{16}$,

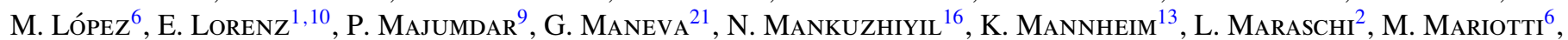
M. Martínez ${ }^{14}$, D. Mazin ${ }^{14}$, M. Meucci ${ }^{11}$, M. Meyer ${ }^{13}$, J. M. Miranda ${ }^{3}$, R. Mirzoyan ${ }^{10}$, H. Miyamoto ${ }^{10}$, J. Moldón ${ }^{12}$, M. Moles $^{18}$, A. Moralejo ${ }^{14}$, D. Nieto ${ }^{3}$, K. Nilsson ${ }^{20}$, J. Ninkovic ${ }^{10}$, N. Otte ${ }^{10,28}$, I. Oya ${ }^{3}$, R. Paoletti ${ }^{11}$, J. M. Paredes ${ }^{12}$, M. Pasanen ${ }^{20}$, D. Pascoli ${ }^{6}$, F. Pauss ${ }^{1}$, R. G. Pegna ${ }^{11}$, M. A. Perez-Torres ${ }^{18}$, M. Persic ${ }^{16,22}$, L. Peruzzo ${ }^{6}$, F. Prada ${ }^{18}$, E. Prandini ${ }^{6}$, N. Puchades ${ }^{14}$, I. ReichardT ${ }^{14}$, W. Rhode ${ }^{4}$, M. Ribó ${ }^{12}$, J. Rico ${ }^{14,23}$, M. Rissi ${ }^{1}$, A. RoberT ${ }^{5}$, S. RÜGamer ${ }^{13}$,

A. Saggion ${ }^{6}$, T. Y. Saito ${ }^{10}$, M. Salvati ${ }^{2}$, M. Sanchez-Conde ${ }^{18}$, K. Satalecka ${ }^{9}$, V. Scalzotto ${ }^{6}$, V. Scapin ${ }^{16}$,

T. Schweizer ${ }^{10}$, M. Shayduk ${ }^{10}$, S. N. Shore ${ }^{24}$, N. Sidro ${ }^{14}$, A. Sierpowska-BartosiK ${ }^{17}$, A. Sillanpä̈̈ ${ }^{20}$, J. Sitarek ${ }^{8,10}$,

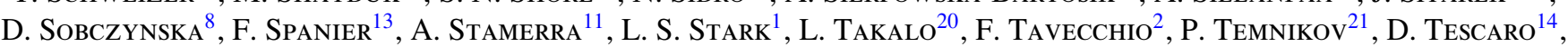

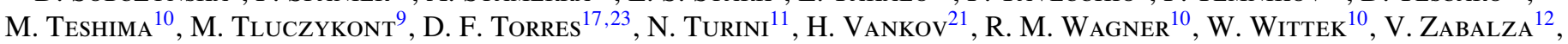
F. ZANDANEL ${ }^{18}$, R. ZANIN ${ }^{14}$, AND J. ZAPATERO ${ }^{5}$

${ }^{1}$ ETH Zurich, CH-8093, Switzerland

2 INAF National Institute for Astrophysics, I-00136 Rome, Italy

${ }^{3}$ Universidad Complutense, E-28040 Madrid, Spain

4 Technische Universität Dortmund, D-44221 Dortmund, Germany

${ }^{5}$ Universitat Autònoma de Barcelona, E-08193 Bellaterra, Spain

${ }^{6}$ Università di Padova and INFN, I-35131 Padova, Italy

${ }^{7}$ Inst. de Astrofísica de Canarias, E-38200 La Laguna, Tenerife, Spain ${ }^{8}$ University of Łódź, PL-90236 Lodz, Poland

${ }^{9}$ Deutsches Elektronen-Synchrotron (DESY), D-15738 Zeuthen, Germany

${ }^{10}$ Max-Planck-Institut für Physik, D-80805 München, Germany

${ }^{11}$ Università di Siena, and INFN Pisa, I-53100 Siena, Italy

12 Universitat de Barcelona (ICC/IEEC), E-08028 Barcelona, Spain

${ }^{13}$ Universität Würzburg, D-97074 Würzburg, Germany

${ }^{14}$ IFAE, Edifici Cn., Campus UAB, E-08193 Bellaterra, Spain

15 Depto. de Astrofisica, Universidad, E-38206 La Laguna, Tenerife, Spain

${ }^{16}$ Università di Udine, and INFN Trieste, I-33100 Udine, Italy

${ }^{17}$ Institut de Cienciès de 1'Espai (IEEC-CSIC), E-08193 Bellaterra, Spain

${ }^{18}$ Inst. de Astrofísica de Andalucia (CSIC), E-18080 Granada, Spain

${ }^{19}$ University of California, Davis, CA-95616-8677, USA

20 Tuorla Observatory, University of Turku, FI-21500 Piikkiö, Finland

${ }^{21}$ Institute for Nuclear Research and Nuclear Energy, BG-1784 Sofia, Bulgaria

22 INAF/Osservatorio Astronomico and INFN, I-34143 Trieste, Italy ${ }^{23}$ ICREA, E-08010 Barcelona, Spain

24 Università di Pisa, and INFN Pisa, I-56126 Pisa, Italy

Received 2009 May 6; accepted 2009 July 2; published 2009 August 10

\section{ABSTRACT}

Based on MAGIC observations from 2007 June to July, we have obtained an integral upper limit to the VHE energy emission of the globular cluster M13 of $F(E>200 \mathrm{GeV})<5.1 \times 10^{-12} \mathrm{~cm}^{-2} \mathrm{~s}^{-1}$, and differential upper limits for $E>140 \mathrm{GeV}$. Those limits allow us to constrain the population of millisecond pulsars within M13 and to test models for acceleration of leptons inside their magnetospheres and surrounding. We conclude that in M13 either millisecond pulsars are fewer than expected or they accelerate leptons less efficiently than predicted.

Key words: gamma rays: observations - globular clusters: individual (M13)

\section{INTRODUCTION}

\footnotetext{
25 Supported by INFN Padova.

${ }^{26}$ Now at Centro de Investigaciones Energéticas, Medioambientales y

Tecnológicas (CIEMAT), E-28040 Madrid, Spain.

27 Deceased.

${ }^{28}$ Now at University of California, Santa Cruz, CA 95064, USA.
}

Globular clusters (GCs) are very interesting sites for probing high energy processes due to their large content of evolved objects. Millisecond pulsars (MSPs) constitute a large fraction of these objects, and it has been estimated that a typical 
massive GC contains of the order of 100 of them (Tavani 1993). Moreover, the largest sample of MSPs discovered up to now in radio observations are located in the GC Ter 5 (23 MSPs) and Tuc 47 (22 MSPs; see e.g., Camilo \& Rasio 2005).

Fluxes of $\mathrm{TeV} \gamma$-rays from GC detectable by current Cherenkov Telescopes have been predicted based on estimates on the population of MSPs and the efficiency of lepton acceleration in their surrounding (see Bednarek \& Sitarek 2007; Venter el al. 2009). These $\gamma$-rays would be produced by accelerated leptons scattering off photons of the microwave background radiation or the thermal emission of an extremely dense cluster of solar mass stars inside the GC. Acceleration of leptons could take place in (1) the shocks within the GC, coming from the collision of the winds of MSPs or (2) the pulsar inner magnetosphere or their wind regions. In addition, $\gamma$-rays in the sub-TeV energy range could also be originated in the inner MSP magnetosphere directly, as it is predicted in the calculations by Bulik et al. (2000) and Harding et al. (2005), or could be produced in the vicinity of radio emitting blocked pulsars (Aharonian et al. 2005; Albert et al. 2006) inside low-mass binary systems (Tavani 1991).

GCs have been observed occasionally by Cherenkov Telescopes to probe for this possible VHE $\gamma$-ray emission. The few experimental results reported in the literature are upper limits on the emission of M13 by the WHIPPLE Collaboration (Hall et al. 2003), M15 by the VERITAS Collaboration (LeBohec et al. 2003), and $\omega$ Centauri by the CANGAROO Collaboration (Kabuki et al. 2007). Very recently, the Fermi LAT has detected high-energy $\gamma$-ray emission $(E>100 \mathrm{MeV})$ from one of the closest and most massive GC, Tuc 47 (Guillemot et al. 2009), and H.E.S.S. has obtained an upper limit of $6.7 \times 10^{-13} \mathrm{ph} \mathrm{cm}^{-2} \mathrm{~s}^{-1}$ for energies $E>800 \mathrm{GeV}$ (Aharonian et al. 2009), but given the possible complexity of the emission in the GeV range, it is not possible to establish any connection between these results. This H.E.S.S. result constrains the magnetic field in the pulsar nebula as a function of the number of MSP in the GC for the model by Venter el al. (2009), and in the efficiency of the rotational energy conversion of MSPs into relativistic leptons for the model by Bednarek \& Sitarek (2007).

In this paper, we report the results of observations with the MAGIC telescope of the globular cluster M13, and we discuss the constraints that our results impose to the population of MSPs and their lepton acceleration efficiency. M13 belongs to the class of normal GCs and has an estimated mass of $6 \times 10^{5} M_{\odot}$. It is located in the Northern sky at a distance of $7 \mathrm{kpc}$. Its core radius is about $\sim 1.6 \mathrm{pc}$, with a half-mass radius of $\sim 3.05 \mathrm{pc}$ (Harris 1999). By now five MSPs have been detected in M13, with periods ranging between 2 and $10 \mathrm{~ms}$. The aforementioned observation of this cluster in search for VHE emission by the WHIPPLE Collaboration (Hall et al. 2003) led to a flux upper limit of $1.08 \times 10^{-11} \mathrm{ph} \mathrm{cm}^{-2} \mathrm{~s}^{-1}$ at energies $E>500 \mathrm{GeV}$.

\section{OBSERVATIONS AND DATA ANALYSIS}

The MAGIC telescope is an Imaging Atmospheric Cherenkov Telescope (IACT) located at the Observatory Roque de los Muchachos on the Canarian Island La Palma. It has an exceptional light detection efficiency provided by the combination of a $17 \mathrm{~m}$ diameter mirror and a pixelized camera composed of 576 high quantum efficiency, hemispherical photomultiplier tubes (PMTs). This allows MAGIC to reach a standard trigger threshold of $\sim 60 \mathrm{GeV}$. For energies above $150 \mathrm{GeV}$, angular and energy resolutions of the telescope are $\sim 0.1$ and $\sim 25 \%$, respectively (see Albert et al. 2008a for further details). Besides this, in 2007 February its data acquisition system was upgraded with multiplexed $2 \mathrm{GHz}$ Flash Analog-Digital converters which improved the timing resolution of the recorded shower images. Accordingly, the sensitivity of MAGIC improved significantly (Aliu et al. 2009) to $1.6 \%$ of the Crab Nebula flux above $270 \mathrm{GeV}$ for $50 \mathrm{hr}$ of observation.

We observed M13 at zenith angles ranging from $8^{\circ}$ to $31^{\circ}$ between June 12 and July 18 of 2007 in a false-source tracking (wobble) mode (Fomin et al. 1994), with two directions at $24^{\prime}$ distance and opposite sides of the source direction. This technique allows for a reliable estimation of the background with no need of extra observation time. The collected data amount to $20.7 \mathrm{hr}$ after rejecting events affected by unstable hardware or environmental conditions. Besides this, events with a collected charge below 300 photoelectrons were rejected in order to maximize the analysis sensitivity. This selection resulted in a sample with a peak energy of $190 \mathrm{GeV}$.

Data analysis was carried out using the standard MAGIC analysis and reconstruction software chain, which proceeds in several steps. Initially, a standard calibration of the PMT signal pulses is performed (Albert et al. 2008b). Then, pixels containing no useful information for the shower image reconstruction are discarded by an image cleaning procedure (Aliu et al. 2009). Afterward event image parameters are calculated (Hillas 1985) using the surviving pixels. In addition to the classical Hillas parameters, two timing parameters are computed, namely, the gradient of the arrival times of the Cherenkov photons along the shower axis and their arrival time spread over the whole shower. The signal-to-noise maximization is achieved using a multidimensional classification procedure based on the Random Forest (RF) method (Albert et al. 2008c), where a hadron likeness measure, the so-called hadronness, is computed for each event based on the image and time parameters. Moreover, a regression RF trained with a Monte Carlo simulated $\gamma$-ray sample is used to estimate the energy on an event-by-event basis. Finally, the angle between the major axis of the shower image ellipse and the source position in the camera, the so-called Alpha angle, is used to select $\gamma$-ray candidates in the direction of the source. To estimate the residual background, the angle Alpha is also computed with respect to a position symmetric to the source position with respect to the camera center. In what follows, this position used to estimate the residual background is referred as the background region.

Main contributions to the systematic error of our analysis are the uncertainties in the atmospheric transmission, the reflectivity of the mirrors (including losses due to surface roughness) and the light catchers, the photon to photoelectron conversion calibration, and the photoelectron collection efficiency in the photomultiplier front end. A detailed discussion of their contribution to the flux uncertainties can be found in Albert et al. (2008a), where they are estimated to add up to $30 \%$ of the measured flux value.

\section{RESULTS}

Figure 1 shows the obtained distribution of the Alpha angle for the source region and the estimated background. It has been obtained for events surviving a hadronness cut tuned to yield an energy-independent $\gamma$-ray selection efficiency of $80 \%$, estimated by means of a Monte Carlo simulation. We define the signal region as the smaller interval in Alpha angle that contains the $80 \%$ of the $\gamma$-rays for each energy bin, estimated using a Monte Carlo simulation. Their lower bounds are at Alpha $=0$ 


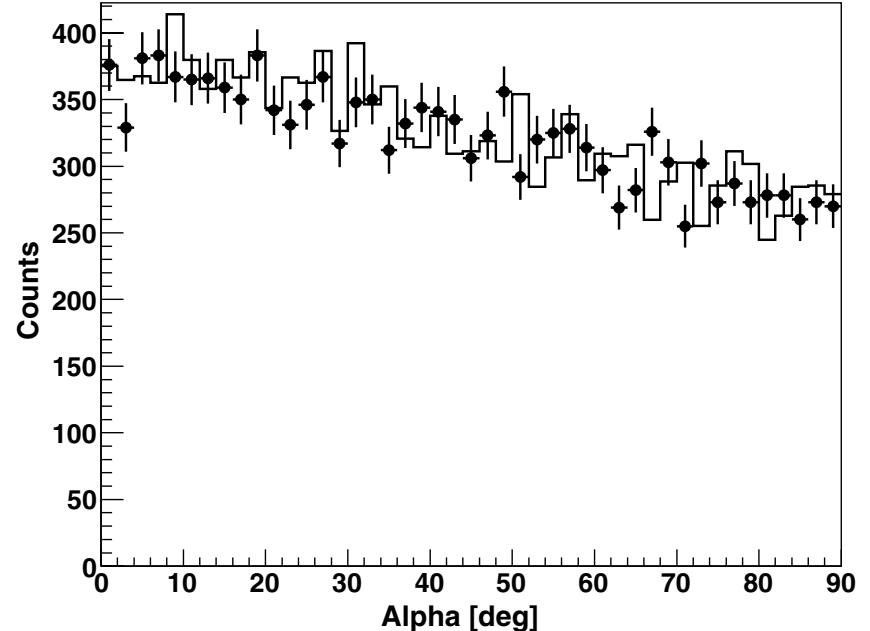

Figure 1. Distribution of Alpha for the selected $\gamma$-ray candidates from the source (black dots) and the background (histogram) regions.

and the upper ones are shown in the second column of Table 1 for each energy bin. We find a total of $-23 \pm 57$ excess events after background subtraction in this signal region for an energy range extending from $140 \mathrm{GeV}$ to $1.1 \mathrm{TeV}$. In addition, a search for signals in a region of $1^{\circ}$ of radius around M13 yields no positive detection. We have obtained upper limits to the VHE flux from M13 for different energy bins, as shown in Table 1. These have been computed using the Rolke method by Rolke et al. (2005) at a $95 \%$ confidence level (CL), and they take into account a $30 \%$ of systematic uncertainties in the flux level. The upper limit to the integral flux for energies above $E=200 \mathrm{GeV}$, assuming a spectral index of 2.6 , is $5.1 \times 10^{-12} \mathrm{~cm}^{-2} \mathrm{~s}^{-1}$.

\section{COMPARISON WITH MODELS}

In Figure 2, we compare our flux upper limits, multiplied by the central value of the range quoted in the first column of Table 1 squared for each energy bin, with the theoretical $\gamma$-ray spectra calculated by Bednarek \& Sitarek (2007). In this model, leptons are injected into the GC volume according to a power-law spectrum, upon acceleration in the shocks produced in the collisions of the pulsar winds of several MSPs. $\gamma$-rays are then produced via Inverse Compton scattering of photons from the microwave background radiation and the thermal radiation arising from the whole GC. Thus, the comparison of our experimental upper limits with the different theoretical gamma-ray spectra allows us to constrain the total power of injected leptons $\left(L_{\mathrm{e}}\right)$. For this, we require the theoretical gammaray spectra to be lower than all the obtained experimental limits, we take into account the light field of the GC (Bednarek $\&$ Sitarek 2007) and assume a distance of 7 pc. The upper limits to $L_{\mathrm{e}}$ are reported in Table 2 for different assumptions

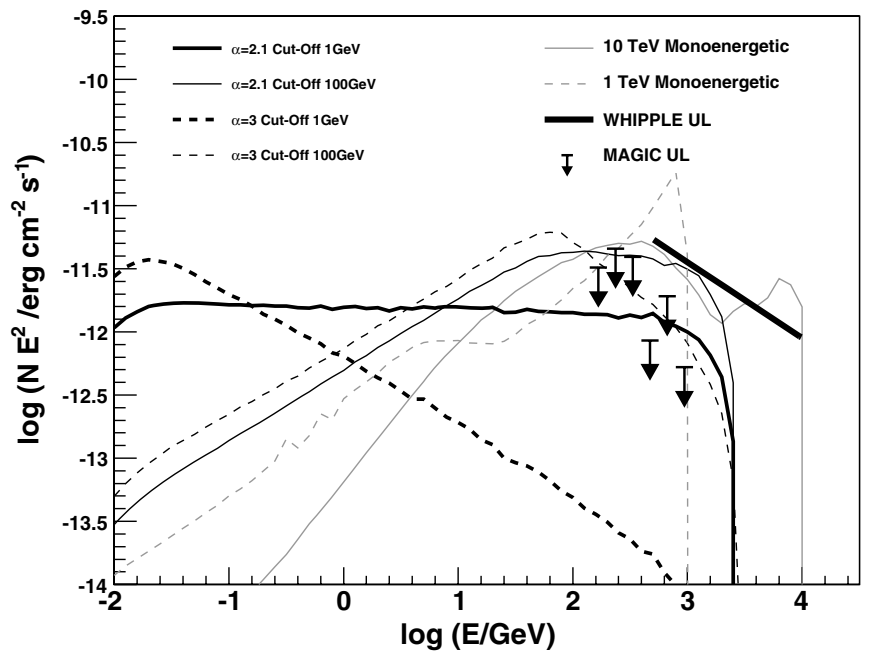

Figure 2. MAGIC $\gamma$-ray flux upper limits for M13 compared with spectra expected for the range of parameters of the model shown in Figures 9 and 10 of Bednarek \& Sitarek (2007). The specific $\gamma$-ray spectra are calculated for lepton upper energy cutoff at $3 \mathrm{TeV}$ and lower energy cutoff at $1 \mathrm{GeV}$ (black thick) and $100 \mathrm{GeV}$ (black thin), and power-law spectral indices of 2.1 (solid) and 3 (dashed). The $\gamma$-ray spectra produced by monoenergetic leptons of $10 \mathrm{TeV}$ and $1 \mathrm{TeV}$ are shown by a gray solid curve and a dashed one, respectively. All calculations are computed assuming the conservative value of 1 for the free parameter of the model $N_{\text {MSP }} \cdot \eta$. The Whipple differential upper limit shown here has been derived from the integral quoted in Hall et al. (2003) assuming a spectral index of 2.6.

of the spectrum of the injected leptons, i.e., for different values of the spectral index $\alpha$ between the minimum energy $E_{\min }$ and the maximum energy defined by the escape of leptons from the shock. In the case of monoenergetic injection of leptons, we consider two different energies, $1 \mathrm{TeV}$ and $10 \mathrm{TeV}$. Assuming characteristic values for the parameters of the MSPs in GCs (surface magnetic field $10^{9} \mathrm{G}$ and rotational period $4 \mathrm{~ms}$ ), we can translate the limits to $L_{\mathrm{e}}$ into limits to the product of the required number of the MSPs in M13 $\left(N_{\mathrm{MSP}}\right)$ times the efficiency of the rotational energy conversion of MSPs into relativistic leptons $(\eta)$, as shown in Table 2. For example, in the case of M13, Tavani (1993) predicts the existence of 100 MSPs. On the other hand, the efficiency of lepton injection from the inner magnetospheres of MSPs has been estimated to be $\eta \sim 0.1$ in terms of the extended polar gap model by Muslinov \& Harding (1997). Therefore, the likely value of the product, $N_{\mathrm{MSP}} \cdot \eta$, should be of the order of $\sim 10$. We show in the corresponding row of Table 2 our estimate of the upper limit to this product for different models of injected spectra of leptons. Moreover, our limits in this product are at the same level than the recently published ones by the H.E.S.S. Collaboration making use of data from 47 Tuc (Aharonian et al. 2009). Figure 3 shows these limits in the $N_{\text {MSP }}, \eta$ plane, such that for each set of model

Table 1

Differential Upper Limits

\begin{tabular}{lccccc}
\hline \hline $\begin{array}{c}\text { Energy Bin } \\
(\mathrm{GeV})\end{array}$ & $\begin{array}{c}\text { Upper Alpha } \\
\text { Cut (deg) }\end{array}$ & Events & $\begin{array}{c}\text { Background } \\
\text { Events }\end{array}$ & $\begin{array}{c}\text { Excess Upper Limit } \\
(95 \% \mathrm{CL})\end{array}$ & $\begin{array}{c}\text { Flux Upper Limit } \\
\left(\mathrm{cm}^{-2} \mathrm{~s}^{-1} \mathrm{TeV}^{-1}\right)\end{array}$ \\
\hline $140-200$ & 8 & 487 & $517 \pm 23$ & 37 & $7.2 \times 10^{-11}$ \\
$200-280$ & 10 & 683 & $681 \pm 27$ & 95 & $5.1 \times 10^{-11}$ \\
$280-400$ & 8 & 254 & $242 \pm 16$ & 75 & $2.2 \times 10^{-11}$ \\
$400-560$ & 6 & 62 & $73 \pm 9$ & 27 & $2.4 \times 10^{-12}$ \\
$560-790$ & 4 & $27 \pm 5$ & 5.8 & $2.7 \times 10^{-12}$ \\
$790-1120$ & 4 & 4 & $5.7 \pm 2.4$ & $3.7 \times 10^{-13}$ \\
\hline
\end{tabular}




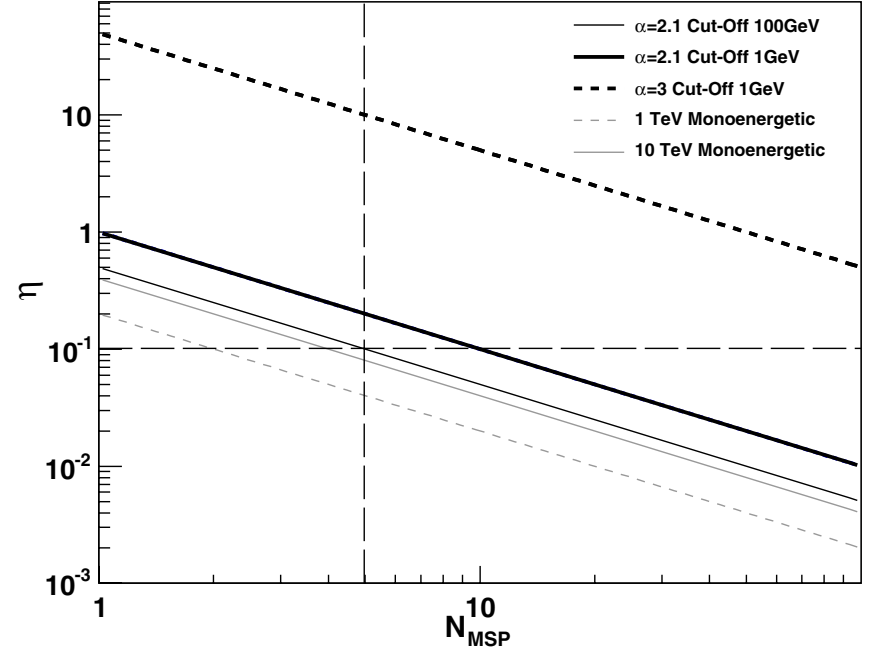

Figure 3. Exclusion contours in the $N_{\mathrm{MSP}}, \eta$ plane for the different models considered in the text. The model with parameters $\alpha=3$ and cutoff at $100 \mathrm{GeV}$ overlaps the $\alpha=2.1$ with cutoff at $1 \mathrm{GeV}$ one. The horizontal and vertical long-dashed black lines show the reference values of $\eta=0.1$ and $N_{\mathrm{MSP}}=5$ respectively.

Table 2

Upper Limits on the Power of Injected Leptons $\left(L_{\mathrm{e}}\right)$ and in $N_{\mathrm{MSP}} \cdot \eta$

\begin{tabular}{lcccccc}
\hline \hline \multicolumn{1}{c}{$E_{\min }$} & $100(\mathrm{GeV})$ & $100(\mathrm{GeV})$ & $1(\mathrm{GeV})$ & $1(\mathrm{GeV})$ & Mono: & Mono: \\
\multicolumn{1}{c}{$\alpha$} & 2.1 & 3.0 & 2.1 & 3.0 & $1(\mathrm{TeV})$ & $10(\mathrm{TeV})$ \\
\hline$L_{\mathrm{e}}$ & 0.6 & 1.0 & 1.0 & 60 & 0.2 & 0.5 \\
$\left(\times 10^{35} \mathrm{erg} \mathrm{s}^{-1}\right)$ & & & & & & \\
$N_{\mathrm{MSP}} \cdot \eta$ & 0.5 & 1.0 & 1.0 & 50 & 0.2 & 0.4 \\
\hline
\end{tabular}

parameters the area above the corresponding curve is excluded at $95 \%$ CL. For most of the considered models, $N_{\mathrm{MSP}} \cdot \eta$ is significantly below $\sim 10$. The only exception is the model with the steep spectrum of leptons which extends down to $1 \mathrm{GeV}$. Note that even if the number of MSPs in M13 is only equal to 5 (as presently observed, Camilo \& Rasio 2005), we can already constrain the acceleration efficiency of leptons to be $\sim 0.1$ in the case of their injection with the hard (spectral index 2.1) and monoenergetic spectrum, respectively (see Figure 3 ).

The $\gamma$-ray spectra produced in the curvature process inside the inner pulsar magnetospheres are predicted to cutoff below $\sim 100 \mathrm{GeV}$ (Bulik et al. 2000; Harding et al. 2005). Thus, they cannot extend to the energy region investigated by our measurement. An inverse Compton $\gamma$-ray component is expected from leptons accelerated in the inner magnetospheres which extends $>100 \mathrm{GeV}$. But its flux is predicted to be at the level of several orders of magnitude below the here presented upper limits for most of the energy range covered by our limits. For example, the Venter el al. (2009) computation of VHE spectrum for GC Tuc 47 and Ter 5 predicts a flux level similar to the ones of the model by Bednarek \& Sitarek (2007) for these pulsars only for narrow-energy band above $E=1 \mathrm{TeV}$.
Therefore, we conclude that the inner magnetosphere $\gamma$-ray emission of MSPs is not likely to be detected by present observations with an analysis threshold of the order of $\sim 100 \mathrm{GeV}$ even from GCs containing hundreds of MSPs.

\section{CONCLUSIONS}

We have obtained the strongest upper limits to date on the VHE $\gamma$-ray flux from the massive globular cluster M13. Our upper limit is $\sim 2$ times lower than the previous limit for VHE energy emission from M13 quoted by WHIPPLE, and extends to energies down to $140 \mathrm{GeV}$. Our upper limits allow us to constrain the population of the MSPs expected in M13 and the acceleration scenarios of leptons by MSPs. Our result strongly suggests that either the number of MSPs in M13 is significantly lower than the estimate of $\sim 100$, or the energy conversion efficiency from MSPs to relativistic leptons is significantly below the value quoted in recent modeling of high energy processes in the magnetospheres of MSPs. Our upper limits regarding Bednarek \& Sitarek (2007) model parameters are the same level than the ones obtained by the H.E.S.S. Collaboration making use of observations of Tuc 47.

We thank the Instituto de Astrofisica de Canarias for the excellent working conditions at the Observatorio del Roque de los Muchachos in La Palma. The support of the German BMBF and MPG, the Italian INFN and Spanish MICINN is gratefully acknowledged. This work was also supported by ETH Research Grant TH 34/043, by the Polish MNiSzW Grant N N203 390834, and by the YIP of the Helmholtz Gemeinschaft.

\section{REFERENCES}

Aharonian, F., et al. 2005, A\&A, 442, 1

Aharonian, F., et al. 2009, A\&A, 499, 273

Albert, J., et al. 2006, Science, 312, 1771

Albert, J., et al. 2008a, ApJ, 674, 1037

Albert, J., et al. 2008b, Nucl. Instrum. Methods A, 594, 407

Albert, J., et al. 2008c, Nucl. Instrum. Methods A, 588, 424

Aliu, E., et al. 2009, Astropart. Phys., 30, 293

Bednarek, W., \& Sitarek, J. 2007, MNRAS, 377, 920 (BS07)

Bulik, T., Rudak, B., \& Dysk, J. 2000, MNRAS, 317, 97

Camilo, F., \& Rasio, F. A. 2005, in ASP Conf. Ser. 328, Binary Radio Pulsars, ed. F. A. Rasio \& I. H. Stairs (San Francisco, CA: ASP), 147

Fomin, V. P., Stepanian, A., Lamb, R. C., Lewis, D. A., Punch, M., \& Weekes, T. C. 1994, Astropart. Phys., 2, 137

Guillemot, L., et al. 2009, in Proc. 44th Rencontres de Moriond, Very High Energy Phenomena in the Universe, in press

Hall, T. A., et al. 2003, ApJ, 583, 853

Harding, A. K., Usov, V. V., \& Muslimov, A. 2005, ApJ, 622, 531

Harris, W. E. 1991, Ap\&SS, 267, 95

Hillas, A. M. 1985, in Proc. 19th Int. Cosmic Ray Conf. (La Jolla), 3, 445

Kabuki, S., et al. 2007, ApJ, 668, 968

LeBohec, S., et al. 2003, in Proc. 28th Int. Cosmic Ray Conf. (Tsukuba), 2521 Muslinov, A. G., \& Harding, A. K. 1997, ApJ, 485, 735

Rolke, W., Lopez, A., Conrad, J., \& James, F. 2005, Nucl. Instrum. Method A, 551,493

Tavani, M. 1991, ApJ, 379, L69

Tavani, M. 1993, ApJ, 407, 135

Venter, C., de Jager, O. C., \& Clapson, A. C. 2009, ApJ, 696, L52 